\title{
South African clinical practice guidelines quality measured with complex and rapid appraisal instruments
}

\author{
Karen Grimmer ${ }^{1,2^{*}}$, Shingai Machingaidze ${ }^{3,4}$, Janine Dizon ${ }^{5,6}$, Tamara Kredo $^{3}$, Quinette Louw ${ }^{2,4}$ \\ and Taryn Young ${ }^{2,5}$
}

\begin{abstract}
Background: Critically appraising the quality of clinical practice guidelines (CPGs) is an essential element of evidence implementation. Critical appraisal considers the quality of CPG construction and reporting processes, and the credibility of the body of evidence underpinning recommendations. To date, the focus on CPG critical appraisal has come from researchers and evaluators, using complex appraisal instruments. Rapid critical appraisal is a relatively new approach for CPGs, which targets busy end-users such as service managers and clinicians. This paper compares the findings of two critical appraisal instruments: a rapid instrument (iCAHE) and a complex instrument (AGREE II). They were applied independently to 16 purposively-sampled, heterogeneous South African CPGs, written for eleven primary health care conditions/health areas. Overall scores, and scores in the two instruments' common domains Scope and Purpose, Stakeholder involvement, Underlying evidence/Rigour of Development, Clarity), were compared using Pearson $r$ correlations and intraclass correlation coefficients. CPGs with differences of $10 \%$ or greater between scores were identified and reasons sought for such differences. The time taken to apply the instruments was recorded.
\end{abstract}

Results: Both instruments identified the generally poor quality of the included CPGs, particularly in Rigour of Development. Correlation and agreement between instrument scores was moderate, and there were no overall significant score differences. Large differences in scores for some CPGs could be explained by differences in instrument construction and focus, and CPG construction. The iCAHE instrument was demonstrably quicker to use than the AGREE II instrument.

Conclusions: Either instrument could be used with confidence to assess the quality of CPGs. The choice of appraisal instrument depends on the needs and time of end-users. Having an alternative (rapid) critical appraisal tool will potentially encourage busy end-users to identify and use good quality CPGs to inform practice decisions.

Keywords: Clinical practice guidelines (CPGs), Rapid appraisal tool, iCAHE checklist, AGREE II, Complex appraisal tool, CPG quality, Reporting standards, Primary health care, South Africa

\section{Background}

Over 20 years ago, Woolf [1-3] described clinical practice guidelines (CPGs) as 'the new reality for medicine'. Research continues into how best to present this 'new reality' to end users in a way that will improve evidence

\footnotetext{
${ }^{*}$ Correspondence: Karen.Grimmer@unisa.edu.au

${ }^{1}$ International Centre for Allied Health Evidence (iCAHE), University

of South Australia, City East Campus, P4-18 North Terrace, Adelaide 5000,

Australia

Full list of author information is available at the end of the article
}

uptake. Whilst there is no one internationally-agreed standard for developing CPGs [4-6], there is a general expectation that CPG recommendations should be transparently based on current best evidence [7-11].

End-users of CPGs are those who put CPG recommendations into operation, such as service managers and healthcare workers 'at the coal face.' These people are rarely engaged in CPG writing [12], however they are usually well aware of the barriers to evidence-uptake [13-16]. These are consistently reported as lack of time, 
money, and knowledge [3, 13-16]. Thus when end-users choose a CPG, they need to be assured that it is of the best possible quality, and that it will efficiently assist them to provide quality care. Service managers and clinicians are busy people, and therefore to assist them in efficiently identifying and using quality CPGs, they require a time-efficient critical appraisal instrument that is comprehensive, simple, robust and efficient.

An Australian team at the International Centre for Allied Health Evidence (iCAHE), University of South Australia, developed and tested a 14 question binaryscored (yes $=1$, no $=0$ ) CPG appraisal instrument, designed specifically for busy end-users [17]. The iCAHE instrument was developed in partnership with service managers, policy-makers and clinicians, and incorporated their perceptions of important elements of CPG quality relevant to their settings. The iCAHE instrument contains 14 questions and provides one overall score (total out of 14). This scoring approach assumes equal weighting for each question, reflecting the views held by the end-users who assisted in its development.

The psychometric properties of the iCAHE instrument were established by comparison with AGREE II (Appraisal of Guideline Research and Evaluation), a complex CPG critical appraisal instrument [7, 18-20]. AGREE II is well-known internationally, and is recommended for assessing CPG quality by the South African Medical Journal $[8,9]$. AGREE II has 23 statements grouped into six domains of Scope and Purpose; Stakeholder Involvement; Rigour of Development; Clarity of Presentation; Applicability; and Editorial Independence. Each statement is scored using a 1-7 scale, with 1 being no agreement and 7 being total agreement. The six domains in AGREE II are intended to be reported separately, and the scoring rubric is not designed to provide an overall quality score $[18,19]$.

The iCAHE and AGREE II instruments share four domains (Scope and Purpose, Stakeholder involvement, Underlying evidence/Rigour, Clarity). The iCAHE instrument also includes three domains not in AGREE II (currency, a summary of findings, and availability), whilst AGREE II includes two domains not in the iCAHE instrument (Applicability, and Independence) (see Table 1). The scores and utility of the iCAHE and AGREE II instruments were compared using six CPGs for mild traumatic brain injury [17]. Overall, the iCAHE and AGREE II scores correlated moderately well (Pearson $r=89 \%)$. Depending on the complexity of CPG layout, the iCAHE instrument took between 5 and 10 min perCPG to apply, whilst the AGREE II instrument scoring per-CPG per-tester took up to an hour.

The South African Guidelines Excellence (SAGE) is a project which aims to improve the quality of South
African primary health care (PHC) CPGs. It is pursuing several research activities, namely identifying, and speaking with, key individuals and groups involved in PHC CPG writing and use in South Africa; determining the quality of current South African PHC CPGs and identifying ways to improve their quality; and building capacity in best practice CPG writing, implementation and evaluation in South African academics, clinicians and policy-makers [21]. The SAGE team recently reported on the quality of 16 purposively-sampled South African CPGs for priority PHC conditions, using AGREE II [22]. These CPGs comprised the most recent versions of seven disease-specific and four integrated multi-disease South African PHC CPGs (see Table 2, reproduced from Machingaidze et al. [22]). The dates of CPG publication ranged from 2002 to 2014. Overall, the quality domains of Rigour of Development, and Editorial Independence had the poorest scores, whilst Scope and Purpose, and Clarity of Presentation generally scored the best. The time taken to score each selected CPGs with AGREE II ranged between 45 and $60 \mathrm{~min}$, depending on CPG layout, comprehensiveness and complexity.

AGREE II was developed for, and has been largely used by, researchers and CPG developers, thus its use may present challenges for time-constrained end-users who have to assess CPG quality by themselves. The iCAHE instrument could be a viable alternative to AGREE II when a rapid overview of CPG quality is required. This paper describes how the iCAHE instrument compares to the AGREE II instrument on a larger set of heterogeneous CPGs.

\section{Methods \\ Data set}

The same 16 purposively-selected South African PHC CPGs reported by Machingaidze et al. [22] were assessed using the iCAHE instrument, and the scores from the two instruments were compared.

\section{Scoring}

The iCAHE instrument was applied by one independent experienced tester whose level of experience was similar to that of the testers who applied the AGREE II instrument [22].

\section{Data management}

To facilitate comparison between instrument scores for each CPG, a percent of possible total (overall) score was calculated for the iCAHE instrument and also from the AGREE II instrument. This approach was previously used when initially validating the iCAHE instrument against AGREE II [17], even though a total AGREE II score is not calculated from the AGREE II domain rubric $[18,19]$. 
Table 1 iCAHE questions mapped against AGREE II domains and their statements

\begin{tabular}{ll}
\hline ICAHE \\
\hline $\begin{array}{l}\text { AGREE II domain 1: scope } \\
\text { and purpose }\end{array} \quad \begin{array}{l}\text { stated? } \\
\text { stare the purpose and target users of the guideline }\end{array}$
\end{tabular}

AGREE II domain 2: stake-

holder involvement

AGREE II domain 3: rigour
of development

iCAHE instrument domain
currency

AGREE II domain 4: clarity of presentation

AGREE II domain 5: applicability

AGREE II domain 6: editorial independence

iCAHE instrument domain: availability

iCAHE instrument domain: summary
Q1. Is the guideline readily available in full text?

Q2. Does the guideline provide a complete reference list?

Q11. Are the developers clearly stated?

Q12. Does the qualifications and expertise of the guideline developers link with the purpose of the guideline and its end users?

Q7. Does the guideline provide an outline of the strategy used to find underlying evidence?

Q8. Does the guideline use a hierarchy to rank the quality of the underlying evidence?

Q9. Does the guideline appraise the quality of the evidence which underpins its recommendations?

Q10. Does the guideline link the hierarchy and quality of underlying evidence to each recommendation?

Q4. Is there a date of completion available?

Q5. Does the guideline provide an anticipated review date?

Q6. Does the guideline provide dates for when literature was included?

Q14. Is the guideline readable and easy to navigate?

Q3. Does the guideline provide a summary of its recommendations?
AGREE II

Q1. The overall objectives of the guideline are specifically described

Q2. The health questions covered by the guideline are specifically described

Q3. The population to whom the guideline is meant to apply is specifically described

Q6. The target users are clearly defined

Q4. The guideline development group includes individuals from all relevant professional groups

Q5. The views and preferences of the target population have been sought

Q7. Systematic methods were used to search for the evidence

Q8. The criteria for selecting the evidence are clearly described

Q9. The strengths and limitations of the body of evidence are clearly described

Q10. The methods for formulating the recommendations are clearly described

Q11. The health benefits, side effects and risks have been considered in formulating the recommendations

Q12. There is an explicit link between the recommendations and the supporting evidence

Q13. The guideline has been eternally reviewed by experts prior to its publication

Q14. A procedure for updating the guideline is provided

Q15. The recommendations are specific and unambiguous

Q16. The different options for management of the condition or health issues are clearly presented

Q17. Key recommendations are easily identifiable

Q18. The guideline describes facilitators and barriers to its application

Q19. The guideline provides advice and/or tools on how the recommendations can be put into practice

Q20. The potential resources implications of applying the recommendations have been considered

Q21. The guideline presents monitoring and/or auditing criteria

Q22. The views of the funding body have not influenced the content of the guideline

Q23. Competing interests of guideline development group members have been recorded and addressed

Adapted from Grimmer et al. [17] 
Table 2 South African CPGs included in this analysis (reproduced from Machingaidze et al. [22])

\begin{tabular}{|c|c|c|c|}
\hline Name & Short name & Publication year & Developer \\
\hline \multicolumn{4}{|l|}{ Disease specific guidelines } \\
\hline $\begin{array}{l}\text { Clinical guidelines for the management of HIV and } \\
\text { AIDS in adults and adolescents }\end{array}$ & Adult HIV & 2010 & National Department of Health \\
\hline Guidelines for the management of HIV in children & Child HIV & 2010 & National Department of Health \\
\hline $\begin{array}{l}\text { Clinical guidelines: PMTCT (prevention of mother-to- } \\
\text { child transmission) }\end{array}$ & РMTCT & 2010 & National Department of Health \\
\hline National tuberculosis management guidelines & Adult TB & 2014 & National Department of Health \\
\hline $\begin{array}{l}\text { Guidelines for the management of tuberculosis in } \\
\text { children }\end{array}$ & Child TB & 2013 & National Department of Health \\
\hline Malaria prevention guidelines & Malaria prevention & 2011 & National Department of Health \\
\hline Malaria treatment guidelines & Malaria treatment & 2010 & National Department of Health \\
\hline \multicolumn{4}{|l|}{ Combination guidelines } \\
\hline $\begin{array}{l}\text { Standard treatment guidelines and essential medicines } \\
\text { list for South Africa }\end{array}$ & $\mathrm{EDL}$ & 2008 & National Department of Health \\
\hline Integrated management of childhood illnesses & $\mathrm{IMCl}$ & 2002 & National Department of Health \\
\hline Guidelines for maternity care in South Africa & Maternal & 2007 & National Department of Health \\
\hline Primary care 101 & PC101 & 2013 & UCT Lung Institute/National Department of Health \\
\hline \multicolumn{4}{|l|}{ Guidelines by professional societies } \\
\hline $\begin{array}{l}\text { Guideline for the management of acute asthma in } \\
\text { adults: } 2013 \text { update }\end{array}$ & Adult asthma & 2013 & South African Thoracic Society \\
\hline $\begin{array}{l}\text { Guideline for the management of acute asthma in } \\
\text { children: } 2013 \text { update }\end{array}$ & Child asthma & 2013 & South African Thoracic Society \\
\hline $\begin{array}{l}\text { Guideline for the management of chronic obstructive } \\
\text { pulmonary disease-2011 update }\end{array}$ & COPD & 2011 & South African Thoracic Society \\
\hline South African hypertension guideline 2011 & Hypertension & 2011 & Southern African Hypertension Society \\
\hline $\begin{array}{l}\text { The } 2012 \text { SEMDSA guideline for the management of } \\
\text { type } 2 \text { diabetes (Revised) }\end{array}$ & Type II diabetes & 2012 & $\begin{array}{l}\text { Society for endocrinology, metabolism and diabetes } \\
\text { of South Africa }\end{array}$ \\
\hline
\end{tabular}

To calculate one percent total score, the individual item responses for all AGREE II statements were applied to the scoring rubric, using a minimum possible score of 23 (calculated as 23 items*1), and a maximum possible score of 171 , calculated as 23 items*7. This score was then reported as a percentage of the possible total.

\section{Analysis}

Correlation between instrument scores was reported as Pearson correlation coefficients (Pearson $r$ ). Significance of instrument score differences was determined using $p$ values from single factor analysis of variance (ANOVA) models, and intraclass correlation coefficients $\left(\mathrm{ICC}_{(2,1)}\right)$ were calculated from the mean square outputs of these ANOVA models. The $\operatorname{ICC}_{(2,1)}$ calculation assumed that the testers were similar to those who might use the instruments in other situations. CPGs with instrument score differences of $>10 \%$ (where positive differences favoured the iCAHE instrument) were identified. The two datasets were:

1. The \% total iCAHE scores and the \% total AGREE II scores for each CPG, using all items in each instru- ment (23 AGREE II statements, 14 iCAHE questions).

2. The $\%$ total scores for only the items in the instruments' common domains (Scope and Purpose, Stakeholder involvement, Rigour of development, Clarity of Presentation). This involved eight iCAHE questions and 17 AGREE II statements. The same process of calculating total AGREE II scores was used as described in the Data management paragraph, however the denominators were $8\left(8^{*} 1\right)$ for iCAHE and 119 (17 items*7) for AGREE II.

The time spent critically appraising the iCAHE instrument was recorded for each CPG, and compared with the time reported by Machingaidze et al. [22]

\section{Results}

Overall CPG quality

Irrespective of whether the iCAHE or AGREE II instrument was used, or the number of questions/statements compared, the overall quality of reporting in the South African PC CPGs was generally poor (See Table 3; Figs. 1 and 2). 


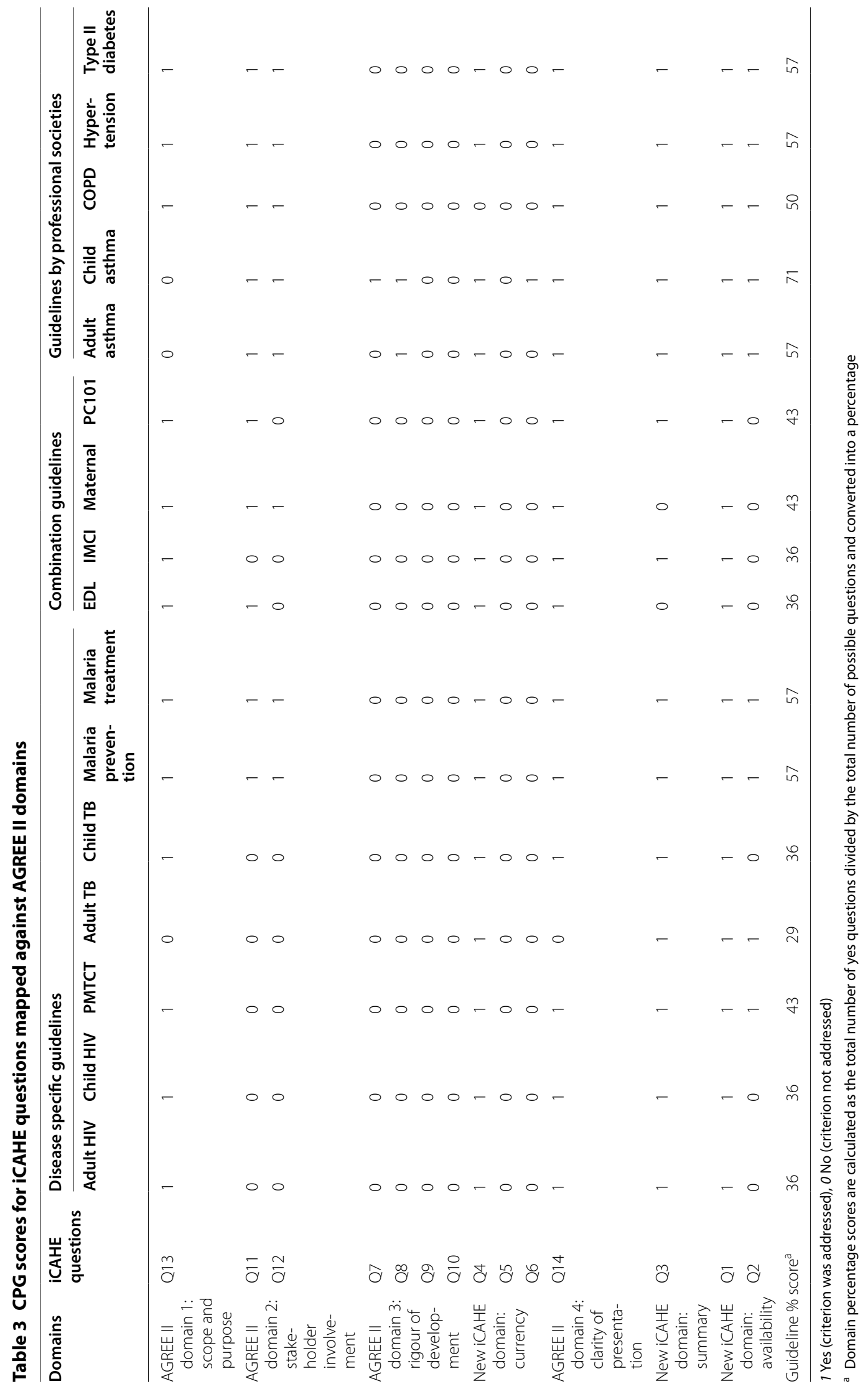




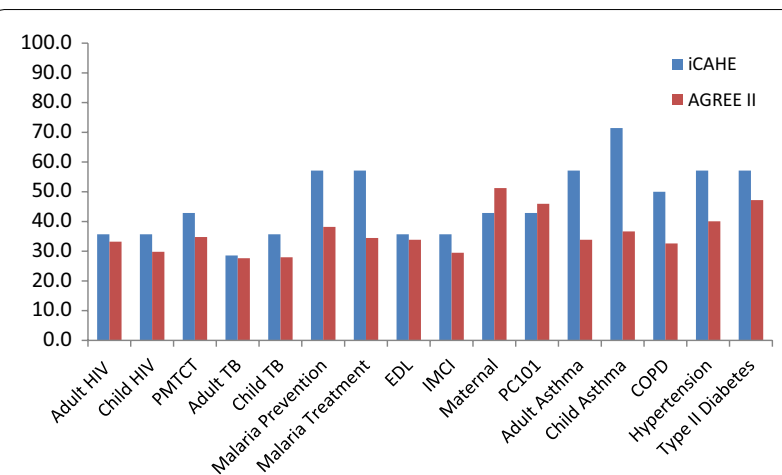

Fig. 1 Analysis 1 findings: comparison of \% of total scores per CPG, including all questions (iCAHE instrument) and statements (AGREE II)

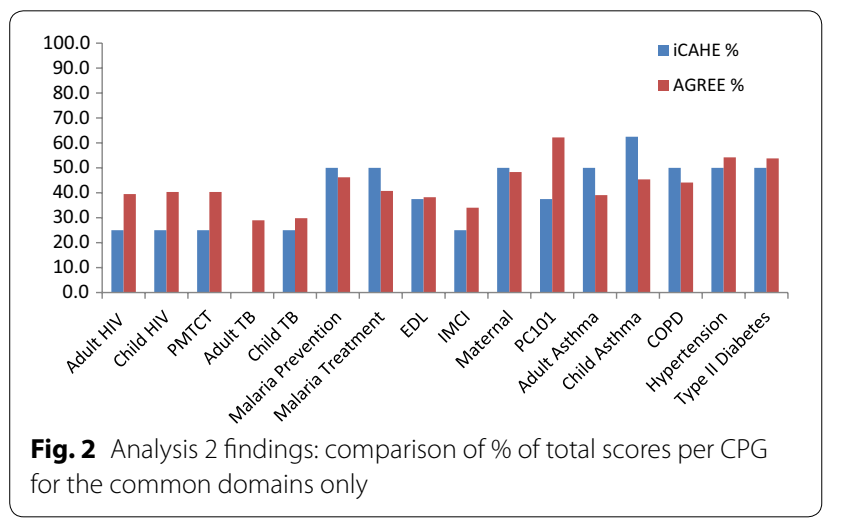

Table 4 reports the findings from analyses 1 and 2 . Comparing analysis 1 with 2, there was an improved correlation between instrument scores for analysis 2 , as well as a stronger $\operatorname{ICC}_{(2,1)}$ score (with a lower, but not significant, $p$ value). There were no significant percentage of score differences overall, from either analysis 1 or 2. However from analysis 1, the large score differences all favoured the iCAHE instrument (see Fig. 1), whilst from analysis 2 , the large score differences mostly favoured the AGREE II instrument (see Fig. 2).

The time to use the iCAHE instrument was 3-5 min per CPG. This mirrored earlier findings on the utility of the iCAHE instrument [17].

\section{Discussion}

This study compare findings from a complex CPG critical appraisal instrument (AGREE II) with a rapid appraisal instrument (iCAHE), on a sizeable sample of heterogeneous country-specific PHC CPGs. Scoring CPG quality is an essential element of evidence implementation $[10,11,13-16]$. Unless end-users have confidence in the quality of the evidence underpinning CPG recommendations, they are unlikely to adopt them. CPGs offer ready access to a 'one-stop-shop' for current best evidencesummaries [1-3]. Irrespective of which critical appraisal instrument was used (rapid or complex), we identified consistent concerns relating to the quality of the selected South African PHC CPGs, particularly in Rigour of

Table 4 Pearson $r$ correlation coefficients, $\operatorname{ICC}_{(2,1)}$ values for agreement and CPGs with disagreements $>10 \%$, listed by order of size of disagreement for analyses 1 and 2

\begin{tabular}{|c|c|c|c|c|}
\hline & $\begin{array}{l}\text { Pearson } r \\
\text { correlation } \\
\text { coefficient }\end{array}$ & $\begin{array}{l}\text { Agreement } \\
\text { expressed as } \\
\text { ICC }_{(2,1)} \text { values }\end{array}$ & $\begin{array}{l}\text { CPGs with } \\
\text { disagreements }>10 \% \\
\text { between instruments }\end{array}$ & $\begin{array}{l}\text { Size of disagreement } \\
\text { (-ve values in favour } \\
\text { of AGREE II) (\%) }\end{array}$ \\
\hline \multirow{7}{*}{$\begin{array}{l}\text { Analysis 1: using all statements in } \\
\text { both instruments }\end{array}$} & \multirow[t]{7}{*}{0.39} & \multirow[t]{7}{*}{$I C C=0.06(p=0.39)$} & (6 of 16) & \\
\hline & & & Child asthma & 34.8 \\
\hline & & & Adult asthma & 23.3 \\
\hline & & & Malaria treatment & 22.7 \\
\hline & & & Malaria prevention & 18.9 \\
\hline & & & COPD & 17.4 \\
\hline & & & Hypertension & 17.1 \\
\hline \multirow{8}{*}{$\begin{array}{l}\text { Analysis 2: using only the state- } \\
\text { ments in the common domains } \\
\text { between the instruments }\end{array}$} & \multirow[t]{8}{*}{0.61} & \multirow[t]{8}{*}{$I C C=0.49(p=0.07)$} & $(7 \circ f 16)$ & \\
\hline & & & Child asthma & 17.1 \\
\hline & & & Adult asthma & 10.9 \\
\hline & & & Adult HIV & -14.5 \\
\hline & & & Child HIV & -15.3 \\
\hline & & & РMTCT & -15.3 \\
\hline & & & PC101 & -24.7 \\
\hline & & & Adult TB & -29.0 \\
\hline
\end{tabular}


Development. This is a similar finding to other studies evaluating South African CPG quality [8, 9].

Analysis 1, which compared the per-CPG total scores derived from the 23 AGREE statements, and the 14 iCAHE questions, demonstrated the modesty of both correlation and agreement. This was attributed to the variability in number and intent, in the two instruments' items. For instance, whilst there were four common domains between instruments, the iCAHE questions included additional domains of Currency, Availability, and Summary, whilst the AGREE II instrument included additional domains of Applicability and Editorial Independence. Comparing differences in total scores, all six CPGs with large percent differences $(>10 \%)$ favoured the iCAHE instrument.

Analysis 2, which compared data from just the four shared domains in the iCAHE and AGREE II instruments, showed improved correlation and agreement, but identified different CPGs with large score differences (with only two of the seven highlighted CPGs favouring the iCAHE instrument). This suggests that the betweeninstrument differences in the number of statements/ questions in the common domains possibly influenced the scoring (8 iCAHE questions in four domains, 17 AGREE II statements in the same four domains). This potentially weighted the overall score in favour of AGREE II.

The shorter time taken to score CPG quality using iCAHE instrument compared with AGREE II reflects the smaller number of items, as well as the utility of the binary-scored iCAHE instrument, where no subjectivity in interpretation is required. In comparison, Machingaidze et al. [22] reported that the AGREE II scores took as much as 10 times longer to compile per CPG, as its use required personal judgement identify a score from 1 to 7 for each statement, and then the application of a scoring rubric per domain. As previously reported [17], this potentially introduces uncertainty in critical appraisal.

\section{Conclusions}

Both appraisal instruments provide standard valid and reliable frameworks for assessment of CPG quality, albeit oriented for different end users. Thus either instrument could be used with confidence to assess the quality of a CPG, and the choice of instrument would depend on the purpose of appraisal, available time and whether additional personnel were available to apply the AGREE II scoring requirements. Having an alternative (rapid) critical appraisal tool will potentially encourage busy endusers who may not currently use complex tools such as
AGREE II, to identify good quality CPGs to inform practice and policy decisions.

\section{Abbreviations \\ CPG(s): clinical practice guideline(s); iCAHE: International Centre for Allied Health Evidence, University of South Australia; AGREE II: Appraisal of Guideline Research and Evaluation; SAGE: South African Guidelines Excellence; PHC: primary health care; ANOVA: analysis of Variance; ICC: intraclass correlation coefficient.}

\section{Authors' contributions}

KG and SM conceptualised the paper, generated the data, undertook the data analysis and drafted the manuscript. JD provided expert input into the manuscript in terms of clinical practice guidelines critical appraisal, data analysis and quality reporting. QL, TK and TY read and commented on drafts of the paper, and brought South African PHC contexts to this work. This paper is not under review elsewhere, and it has not been previously published. We look forward to the reviewers' comments in due course. All authors read and approved the final manuscript.

\section{Author details}

${ }^{1}$ International Centre for Allied Health Evidence (iCAHE), University of South Australia, City East Campus, P4-18 North Terrace, Adelaide 5000, Australia.

${ }^{2}$ Department of Physiotherapy, Faculty of Medicine and Health Sciences, Stellenbosch University, Francie van Zijl Drive, Tygerberg, Cape Town 7505, South Africa. ${ }^{3}$ European and Developing Countries Clinical Trial Partnership (EDCTP), Francie van Zijl Drive, Parow Valley, Cape Town 7505 , South Africa. ${ }^{4}$ South African Cochrane Centre (SACC), South African Medical Research Council, Francie van Zijl Drive, Parow Valley, Cape Town 7505, South Africa. ${ }^{5}$ Centre for Evidence-Based Health Care (CEBHC), Faculty of Medicine and Health Sciences, Stellenbosch University, Francie van Zijl Drive, Tygerberg, Cape Town 7505, South Africa. ${ }^{6}$ Center for Health Research and Movement Science, University of Santo Tomas, Espana, 1018 Manila, Philippines.

\section{Acknowledgements}

There are no acknowledgements to individuals who worked on this project but did not meet authorship requirements. All researchers on the project are named as authors.

\section{Competing interests}

The authors declare that they have no competing interests.

\section{Funding source}

This research and the publication thereof is the result of funding provided by the South African Medical Research Council in terms of the MRC's Flagships Awards Project SAMRC-RFA-IFSP-01-2013/ SAGE. SM worked full time on this project as the project manager, and JD is a post-doctoral student partly funded by this grant. KG, QL, and TY were funded between 4 and $20 \mathrm{~h}$ per week by the project grant to research specific aspects. TK is the Deputy Director, Cochrane South Africa. QL and TY hold professorial appointments at Stellenbosch University, Cape Town, South Africa, and KG holds a professorial position at University of South Australia, Australia, and is a Professor Extraordinaire at Stellenbosch University, South Africa. The funding body was totally independent of the conduct of this project, including how and where its findings are reported. The funding body only has requirements for six-monthly progress reports.

Received: 3 January 2016 Accepted: 19 April 2016

Published online: 27 April 2016

References

1. Woolf SA. Practice guidelines: a new reality in medicine. I. Recent developments. Arch Int Med. 1990;150:1811. 
2. Woolf SA. Practice guidelines: a new reality in medicine. II. Methods of developing guidelines. Arch Int Med. 1992;152:946.

3. Woolf SA. Practice guidelines: a new reality in medicine. III. Impact on patient care. Arch Int Med. 1993;153:2646.

4. Qaseem A, Forland F, Macbeth F, OllenschläTger G, Phillips S, van der Wees P. Guidelines International Network: toward International Standards for Clinical Practice Guidelines. Ann Intern Med. 2012;156:525-31.

5. Graham R, Mancher M, Wolman DM, Greenfield S, Steinberg E, editors. Clinical practice guidelines we can trust. Institute of Medicine (IOM), Committee on Standards for Developing Trustworthy Clinical Practice Guidelines, Board on Health Care Services. Washington: The National Academies Press; 2011.

6. Schünemann HJ, Wiercioch W, Etxeandia I, et al. Guidelines 2.0: systematic development of a comprehensive checklist for a successful guideline enterprise. CAMJ. 2014;186(3):E123-42.

7. Semlitsch T, Blank WA, Kopp IB, Siering U, Siebenhofer A. Evaluating guidelines - a review of key quality criteria. Dtsch Arztebl Int. 2015:112:471-8.

8. Wiseman R, Cohen K, Gray A, Jamaloodien K, Kredo T, et al. AGREE to disagree: critical appraisal and the publication of practice guidelines. SAMJ. 2014;104(5):345-6.

9. Kredo T, Gerritsen A, van Heerden J, Conway S, Siegfried N. Clinical practice guidelines within the Southern African development community: a descriptive study of the quality of quideline development and concordance with best evidence for five priority diseases. Health Res Pol Sys. 2012;10:1

10. Alonso-Coello P, Irfan A, Sola I, Gich I, Delgado-Noguera M, Rigau D, Tort $\mathrm{S}$, Bonfill X, Burgers J, Schunemann $\mathrm{H}$. The quality of clinical practice guidelines over the last two decades: a systematic review of guideline appraisal studies. Qual Saf Health Care. 2010;19(6):e58.

11. Wimpenny P, van Zelm R. Appraising and comparing pressure ulcer guidelines. Worldviews Evid-Based Nurs. 2007:4(1):40-50.

12. Hirsh J, Guyatt G. Clinical experts or methodologists to write clinical guidelines? Lancet. 2009;374(9686):273-5.
13. Grol R, Wensing M. What drives change? Barriers to and incentives for achieving evidence-based practice. Med J Aust. 2004;15(180):57-60.

14. Grimmer K, Lekkas P, Nyland L, Young A, Kumar S. Perspectives on research evidence and clinical practice: a survey of Australian physiotherapists. PRI. 2007;12(3):147-61.

15. Metcalfe C, Lewin R, Wisher S, Perry S, Bannigan K. Klaber Moffett J. Barriers to implementing the evidence base in four NHS therapies. Physiotherapy. 2001;87:433-40.

16. Parahoo K. Barriers to, and facilitators of, research utilisation among nurses in Northern Ireland. J Adv Nurs. 2000;31:89-98.

17. Grimmer K, Dizon J, Milanese S, King E, Beaton K, Thorpe O, Lizarondo L, Luker J, Kumar S, Machokta Z. Efficient clinical evaluation of guideline quality: development and testing of a new tool. BMC Res Notes. 2014;14:63.

18. AGREE Collaboration. Development and validation of an international appraisal instrument for assessing the quality of clinical practice guidelines: the AGREE project. Qual Saf Health Care. 2003;12:18-23.

19. Brouwers M, Kho ME, Browman GP, Burgers JS, Cluzeau F, Feder G, Fervers B, Graham ID, Grimshaw J, Hanna S, Littlejohns P, Makarski J, Zitzelsberger $L$, for the AGREE Next Steps Consortium. AGREE II: Advancing guideline development, reporting and evaluation in healthcare. Can Med Assoc J. 2010;182:E839-42.

20. MacDermid JC, Brooks D, Solway S, Switzer-McIntyre S, Brosseau L, Graham ID. Reliability and validity of the AGREE instrument used by physical therapists in assessment of clinical practice guidelines. BMC Health Serv Res. 2005;5:18.

21. Machingaidze S, Kredo T, Young T, Louw Q, Grimmer K. South African Guidelines Excellence (SAGE): Clinical Practice Guidelines-Quality and Credibility. SAMJ. 2015;105(9):743-5.

22. Machingaidze S, Zani B, Abrams A, Durao S, Louw Q et al. Quality and reporting standards of South African primary care clinical practice guidelines. J Clin Epi. 2016 (in press).

\section{Submit your next manuscript to BioMed Central and we will help you at every step:}

- We accept pre-submission inquiries

- Our selector tool helps you to find the most relevant journal

- We provide round the clock customer support

- Convenient online submission

- Thorough peer review

- Inclusion in PubMed and all major indexing services

- Maximum visibility for your research

Submit your manuscript at www.biomedcentral.com/submit
() Biomed Central 